\title{
A PRECISE CALCULATION OF THE FEIGENBAUM CONSTANTS
}

\author{
KEITH BRIGGS
}

\begin{abstract}
The Feigenbaum constants arise in the theory of iteration of real functions. We calculate here to high precision the constants $\alpha$ and $\delta$ associated with period-doubling bifurcations for maps with a single maximum of order $z$, for $2 \leq z \leq 12$. Multiple-precision floating-point techniques are used to find a solution of Feigenbaum's functional equation, and hence the constants.
\end{abstract}

\section{HISTORY}

Consider the iteration of the function

$$
f_{\mu, z}(x)=1-\mu|x|^{z}, \quad z>0 ;
$$

that is, the sequence

$$
x_{i+1}=f_{\mu, z}\left(x_{i}\right), \quad i=1,2, \ldots ; \quad x_{0}=0 .
$$

In 1979 Feigenbaum [8] observed that there exist bifurcations in the set of limit points of (2) (that is, in the set of all points which are the limit of some infinite subsequence) as the parameter $\mu$ is increased for fixed $z$. Roughly speaking, if the sequence (2) is asymptotically periodic with period $p$ for a particular parameter value $\mu$ (that is, there exists a stable $p$-cycle), then as $\mu$ is increased, the period will be observed to double, so that a stable $2 p$-cycle appears. We denote the critical $\mu$-value at which the $2^{j}$ cycle first appears by $\mu_{j}$.

Feigenbaum also conjectured that there exist certain "universal" scaling constants associated with these bifurcations. Specifically,

$$
\delta_{z}=\lim _{j \rightarrow \infty} \frac{\mu_{j}-\mu_{j-1}}{\mu_{j+1}-\mu_{j}}
$$

exists, and $\delta_{2}$ is about 4.669. Similarly, if $d_{j}$ is the value of the nearest cycle element to 0 in the $2^{j}$ cycle, then

$$
\alpha_{z}=\lim _{j \rightarrow \infty} \frac{d_{j}}{d_{j+1}}
$$

exists, and $\alpha_{2}$ is about -2.503 .

Received November 22, 1989; revised September 10, 1990.

1980 Mathematics Subject Classification (1985 Revision). Primary 11Y60, 26A18, 39A10, $65 \mathrm{Q} 05$. 
The conjecture for the case $z=2$ was proven by Lanford in 1982 [11], and for $z<14$ by Epstein in 1985 [7].

Some numerical results in the literature are given in Table 1. (Note that most authors quote $|\alpha|$.)

\section{TABLE 1}

\begin{tabular}{cccc} 
Reference & $z$ & \multicolumn{1}{c}{$\alpha$} & \multicolumn{1}{c}{$\delta$} \\
{$[8]$} & 2 & -2.502907876 & 4.6692 \\
{$[9]$} & 2 & 2.50290787509589284 & 4.6692016091029909 \\
{$[4]$} & 2 & 2.502907875095892822283902873 & 4.6692016091029906718532038 \\
& & & \\
{$[13]$} & 2 & 2.5029078 & 4.6692016 \\
& 4 & 1.690 & 7.29 \\
& 6 & 1.467 & 9.30 \\
& 8 & 1.358 & 10.948 \\
& 10 & 1.292 & 12.37 \\
{$[5]$} & 2 & 2.50 & \\
& 3 & 1.93 & 4.67 \\
& 4 & 1.69 & 6.08 \\
& 5 & 1.56 & 7.29 \\
& 7 & 1.41 & 8.35 \\
& 10 & 1.29 & 10.2 \\
& & & 12.3
\end{tabular}

Some examples of physical systems in which $\alpha$ and $\delta$ are relevant are described in [2]. Despite the theoretical and applied interest of these numbers, little is known about them, for example whether they satisfy any simple algebraic relations. On the question of the limits as $z$ tends to $\infty$ of $\alpha_{z}$ and $\delta_{z}$, see [6].

We propose here to evaluate $\alpha$ and $\delta$ to high precision for various $z$, in order to provide data for testing conjectures concerning these numbers.

\section{METHOD}

Calculating $\delta$ directly from the definition is impractical because it would involve finding high iterates of $f$, which are subject to accumulation of roundoff error, making it difficult to locate the bifurcation values $\mu_{j}$ accurately.

A practical algorithm for $\delta$ was described in [3]. However, this is suitable for low precision only, owing to its slow convergence. Another method for $\delta$ was proposed in [12], but has the same drawback. It seems that the original method of Feigenbaum [8] is still the best when high precision is desired. We briefly describe this method, which is justified in [8].

One defines an operator $T$, acting on functions $g: \mathbb{R} \rightarrow \mathbb{R}$, by

$$
[T g](x)=\{g(g(g(1) x))\} / g(1) .
$$


If we find an even real analytic function invariant under $T$, with $g(0)=1$, then $\alpha$ is determined by $\alpha=1 / g(1)$.

The numerical method proceeds by approximating $g$ by the form

$$
g(x)=1+\sum_{i=1}^{n} g_{i}|x|^{z i}
$$

An approximate fixed point of $T$ can then be found by a collocation method. We require (5) to be satisfied at $n$ points $x_{j}$ in the interval $(0,1]$, and solve the resulting $n$ nonlinear equations by an $n$-dimensional Newton iteration. Thus we require (for $j=1, \ldots, n$ )

$$
\begin{aligned}
\left(1+\sum_{i=1}^{n} g_{i}\right)\left(1+\sum_{i=1}^{n} g_{i}\left|x_{j}\right|^{z i}\right) \\
\quad-1-\left.\left.\sum_{i=1}^{n} g_{i}\left|1+\sum_{i=1}^{n} g_{i}\right|\left(1+\sum_{i=1}^{n} g_{i}\right) x_{j}\right|^{z i}\right|^{z i}=0
\end{aligned}
$$

If we call the left side of this equation $f_{j}$, the Newton iteration requires the inversion of the Jacobian matrix $\partial f_{j} / \partial g_{i}$. This is the major part of the computational task. For the smaller $z$-values, it was found that the initial approximation to the $g$-coefficients was not critical, but for the larger $z$-values some trial and error was necessary before convergence was obtained. For $z$ greater than 12, all initial approximations tried produced divergence of the Newton iteration. However, it is probable that a solution to (7) does exist for all $z$.

Feigenbaum has shown [8] that the constant $\delta_{z}$ is the largest eigenvalue of the local linearization of $T$ about the fixed point function $g$ found above. A simple calculation shows that this operator $L$ is given by

$$
[L f](x)=-\alpha f(g(x / \alpha))-\alpha g^{\prime}(g(x / \alpha)) f(x / \alpha) .
$$

Once the approximate fixed point $g$ has been found, one may construct a finitedimensional matrix approximating $L$ by a method similar to that used above. That is, one evaluates the right side of (8) at the $n$ nodes $x_{i}$. The largest eigenvalue of the matrix can then easily be found by the power method [10, $\S 7.3]$.

\section{RESULTS}

We have implemented the above scheme with arbitrary-precision floatingpoint arithmetic, using the methods described by Brent [1]. The choice of the points $x_{i}$ was found to be not critical, linear spacing being adequate for small $z$. However, the nonlinear spacing $x_{i}=(i / n)^{1 / z} \quad(i=1, \ldots, n)$ produced more stable results for the higher $z$-values, and was therefore used for all the 
results quoted. It is observed that the $g$-coefficients decrease rapidly in magnitude; for example for $z=2,\left|g_{i}\right|$ is about $10^{-i}$. This gives a guide to the value of $n$ needed; since $\alpha$ is $1 / g(1)$, we must set $n$ about equal to the number of decimals desired for $\alpha$, and preferably greater. We first found $\alpha$ and $\delta$ for $z=2, \ldots, 12$ with $n=75$ and a working precision of 150 decimal places. We then repeated all calculations with $n=100$ and a working precision of 200 decimal places. The results given in Table 2 show as many digits as agree between the two calculations. Thus, it is probable that all quoted digits are correct.

\section{TABLE 2}

Feigenbaum's $\alpha$ and $\delta$ for $z=2,3, \ldots, 12$

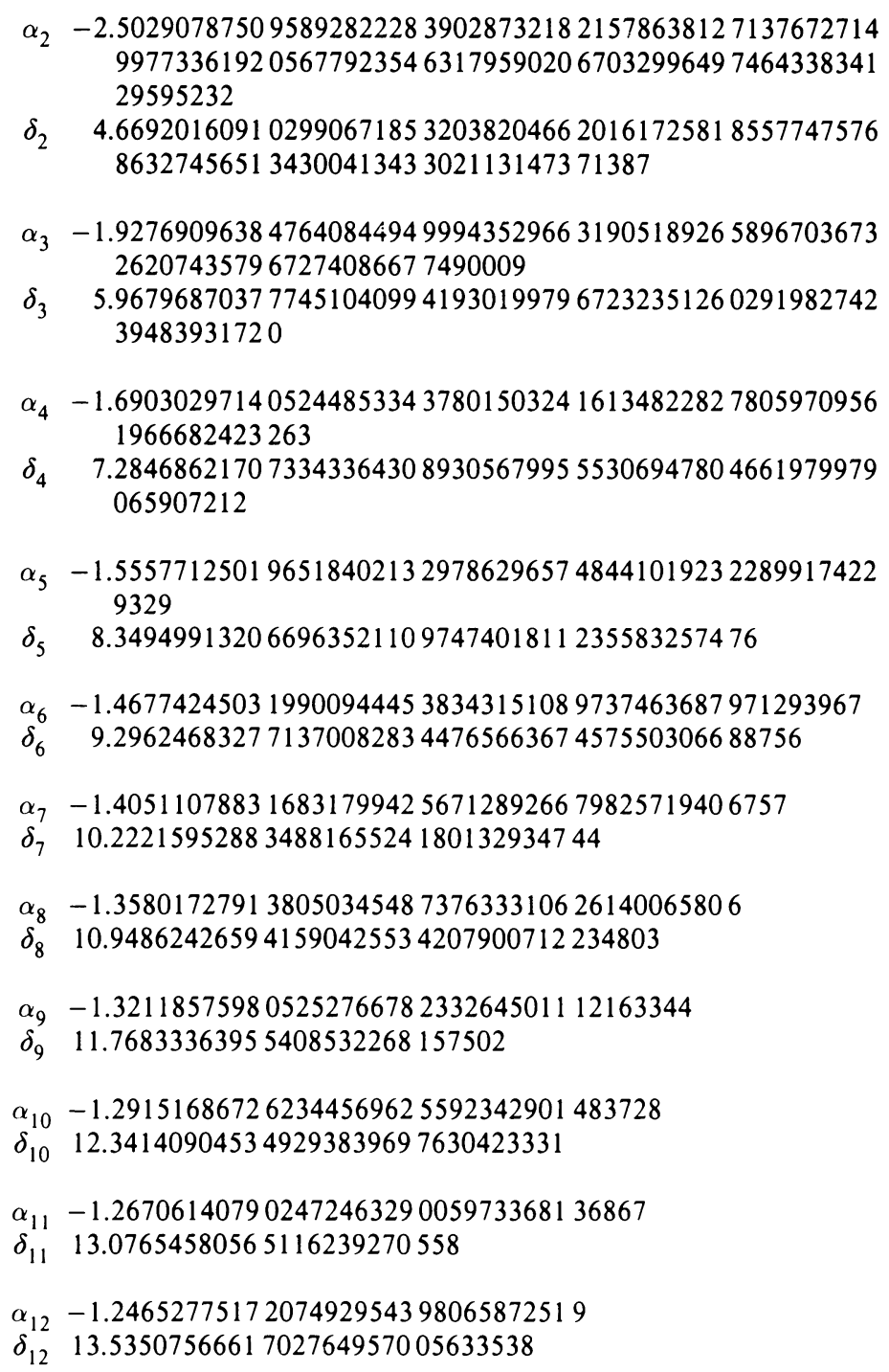




\section{BIBLIOGRAPHY}

1. R. P. Brent, A Fortran multiple-precision arithmetic package, ACM Trans. Math. Software 4 (1978), 57-70.

2. K. M. Briggs, Simple experiments in chaotic dynamics, Amer. J. Phys. 55 (1987), 10831089.

3. $\ldots$, How to calculate the Feigenbaum constants on your PC, Austral. Math. Soc. Gazette 16 (1989), 89-92.

4. F. Christiansen, P. Cvitanović and H. H. Rugh, The spectrum of the period-doubling operator in terms of cycles, J. Phys. A 23 (1990), L713-L717.

5. R. Delbourgo and B. G. Kenny, Universality relations, Phys. Rev. A 33 (1986), 3292-3302.

6. J.-P. Eckmann and P. Wittwer, Computer methods and Borel summability applied to Feigenbaum's equation, Springer-Verlag, 1985.

7. H. Epstein, New proofs of the existence of the Feigenbaum functions, Report No. IHES/P/85/55, Inst. Hautes Études Sci., 1985.

8. M. J. Feigenbaum, The universal metric properties of nonlinear transformations, J. Statist. Phys. 21 (1979), 669-706.

9. __ The metric universal properties of period doubling bifurcations and the spectrum for a route to turbulence, Ann. New York Acad. Sci. 357 (1980), 330-336.

10. G. H. Golub and C. F. Van Loan, Matrix computations, 2nd ed., The Johns Hopkins Univ. Press, Baltimore, 1989.

11. O. E. Lanford III, A computer-assisted proof of the Feigenbaum conjectures, Bull. Amer. Math. Soc. 6 (1982), 427-434.

12. J. B. McGuire and C. J. Thompson, Asymptotic properties of sequences of iterates of nonlinear transformations, J. Statist. Phys. 27 (1982), 183-200.

13. A. Mo and P. C. Hemmer, Iterative properties of nonquadratic one-dimensional maps, Phys. Scripta 29 (1984), 296-302.

14. C. J. Thompson and J. B. McGuire, Asymptotic and essentially singular solutions of the Feigenbaum equation, J. Statist. Phys. 51 (1988), 991-1007.

Department of Mathematics, La Trobe University, Bundoora, Victoria 3083, AusTRALIA

Current address: Department of Mathematics, University of Melbourne, Parkville 3052, Australia 